\title{
ANDES

\section{Encuesta para cuidadores de personas del espectro autista en Chile: primeras preocupaciones, edad del diagnóstico y características clínicas}

\author{
Survey for caregivers of people in the autism spectrum in Chile: first concerns, \\ age of diagnosis and clinical characteristics
}

\author{
Ricardo García ${ }^{\mathrm{a}, \mathrm{b}, \mathrm{c}}$, Matías Irarrázavala, ${ }^{\mathrm{a}, \mathrm{d}}$, Isabel López ${ }^{\mathrm{a}, \mathrm{e}}$, Sofía Riesle $^{\mathrm{a}}$, Marcia Cabezas ${ }^{\mathrm{a}, \mathrm{b}}$, Andrea Moyano $^{\mathrm{a}}$
}

\author{
aPrograma de Trastornos del Espectro Autista, Clínica Psiquiátrica, Facultad de Medicina, Universidad de Chile. Santiago, Chile. \\ bDepartamento de Psiquiatría Infantil y Adolescente, Clínica Las Condes. Santiago, Chile. \\ 'Red de Espectro Autista Latinoamericano (REAL)* \\ 'Ministerio de Salud, Gobierno de Chile. Santiago, Chile. \\ eDepartamento de Neurología Pediátrica, Clínica Las Condes. Santiago, Chile.
}

\begin{abstract}
*Este trabajo forma parte de un Proyecto más amplio del Grupo REAL (Red de Espectro Autista Latinoamericano), con la participación de los siguientes investigadores: G. Garrido, Universidad de la República, Montevideo, Uruguay. D, Valdés, FLACSO, UBA, Buenos Aires, Argentina, C. Paula, Universidade Federal de Sao Paulo, Sao Paulo, Brazil. A. Rosoli, OEl, Santo Domingo, República Dominicana, S. Cukier, PANAACEA, Buenos Aires, Argentina. C. Montiel-Nava, Universidad Latina Panamá and INDICAST, Panamá A. Rattazzi, PANAACEA, Buenos Aires, Argentina.
\end{abstract}

Recibido: 7 de mayo de 2020; Aceptado: 13 de septiembre de 2020

¿Qué se sabe del tema que trata este estudio?

Hasta el momento del envío de este trabajo para su publicación, no existen antecedentes de reportes de la percepción de las preocupaciones y la edad de diagnóstico de las personas del espectro autista de parte de sus cuidadores.
¿Qué aporta este estudio a lo ya conocido?

Devela la gran brecha entre edad de primeras preocupaciones de los padres y el diagnóstico médico de TEA, lo que significa una pérdida crítica de oportunidades de tratamiento, lo que puede poner en riesgo las posibilidades de un mejor pronóstico futuro.

\section{Resumen}

Los Trastornos del Espectro Autista (TEA) son desórdenes del neurodesarrollo de prevalencia en aumento. Las personas TEA tienen múltiples necesidades de salud, educación y comunitarias. En Chile existe escasa información al respecto. Objetivo: Conocer características demográficas, clínicas, primeras preocupaciones de sus cuidadores y la edad de diagnóstico de personas TEA. Pacientes y Método: Participaron padres/cuidadores de personas TEA, quienes respondieron la Encuesta de $\mathrm{Ne}$ cesidades de Cuidadores, desarrollada por Autism Speaks para este propósito específico y traducida al
Palabras clave:

Trastornos del Espectro Autista; Autismo; Cuidadores; Encuesta de Salud; Primeras Preocupaciones; Edad de Diagnóstico; Diagnóstico Temprano; Chile; América Latina

Correspondencia:

Ricardo García

ricardo.garcia.sepulveda@gmail.com 
español. La encuesta comprende 4 secciones: información demográfica, características de la persona TEA, utilización pasada y presente de Servicios de Salud y Educación y percepciones de Padres/Cuidadores en cuanto a satisfacción, impacto, estigma, y calidad de vida. Aquí se reporta información proveniente de las dos primeras secciones. Resultados: Participaron 291 cuidadores ( $86 \%$ madres) de 291 personas TEA, 89\% varones, de entre 1-40 años (X: 10,4 DE: 6,1). La edad promedio de primeras preocupaciones fue 29,2 m (DE: 23,8) y las principales fueron: dificultades en interacción (79,4\%), respuesta inusual a estímulos sensoriales $(69,8 \%)$, dificultades de comportamiento $(65,3 \%)$, gestos/ movimientos inusuales $(64,3 \%)$, falta de contacto visual $(63,6 \%)$. La edad promedio de diagnóstico fue $58 \mathrm{~m}$ (DE: 36,5), con retraso promedio del diagnóstico de $29 \mathrm{~m}$. El diagnóstico fue realizado por neurólogos pediátricos (44,7\%), psiquiatras infantiles (19,2\%), pediatras (5,5\%). Las comorbilidades más frecuentes fueron compromiso de lenguaje, déficit cognitivo y problemas conductuales. Conclusiones: La tardía edad de diagnóstico y la gran brecha entre edad de primeras preocupaciones y diagnóstico de TEA representan una pérdida crítica de oportunidades de tratamiento, lo que puede poner en riesgo las posibilidades de un mejor pronóstico futuro.

\begin{abstract}
Autism Spectrum Disorders (ASD) are neurodevelopmental disorders of increasing prevalence. People with ASD have multiple health, education, and community needs, yet there is little information about their situation in Chile. Objective: To learn about the demographic and clinical characteristics, caregiver's first concerns, and age of diagnosis of ASD individuals. Patients and Method: Participants were parents/caregivers of ASD persons, who answered the Caregiver Needs Survey, developed by Autism Speaks specifically for this purpose, and translated into Spanish. The survey is comprised of 4 sections: demographic information, characteristics of the ASD individual, past and present use of Health and Education Services, and parents/caregivers' perceptions of satisfaction, impact, stigma, and quality of life. Data from the first two sections are reported in this paper. Results: The survey was answered by 291 caregivers ( $86 \%$ mothers) of 291 mostly male ASDs (89\%), aged between 1-40 years (X: 10.4 SD: 6.1). The average age of parents' first concerns was $29.2 \mathrm{~m}$ (SD: 23.8) where the main ones were: interaction difficulties $(79.4 \%)$, unusual response to sensory stimuli $(69.8 \%)$, behavioral problems $(65.3 \%)$, unusual gestures/movements $(64.3 \%)$, and lack of eye contact $(63.6 \%)$. The average age of diagnosis was $58 \mathrm{~m}$ (SD: 36.5 ), with an average delay of diagnosis of $29 \mathrm{~m}$. The diagnosis was most frequently made by pediatric neurologists $(44.7 \%)$, child psychiatrists $(19.2 \%)$, and pediatricians (5.5\%). The most frequent comorbidities were language impairment, cognitive deficit, and behavioral problems. Conclusions: The late age of diagnosis of ASD and the large gap between the age of first concerns and diagnosis, represent a critical loss of treatment opportunities and jeopardize the chances of a better long-term outcome.
\end{abstract}

Keywords:

Autism Spectrum

Disorders;

Autism;

Caregivers;

Health Survey;

First Concerns;

Age of Onset;

Age of Diagnosis;

Early Diagnosis;

Chile; Latin America

\section{Introducción}

El aumento de prevalencia de Trastornos del Espectro Autista (TEA), con cifras actuales alarmantes de hasta 1:54 niños de 8 años ${ }^{1}$ es un fenómeno que, independientemente de factores culturales, socioeconómicos o étnicos, afecta a todas las regiones del mundo ${ }^{2,3}$. Existe evidencia contundente acerca de la relevancia de la detección temprana, en tanto intervenciones intensivas, apropiadas y oportunas se traducen en mejoras significativas de habilidades cognitivas y de lenguaje, cambios en las trayectorias de desarrollo y una consiguiente reducción de los déficit ${ }^{4,5}$.

Muchos factores contribuyen al retraso en el diagnóstico, entre ellos la severidad de los síntomas, el nivel socio-económico, las preocupaciones de los padres en cuanto a las manifestaciones iniciales y el acceso de la familia a sistemas de salud y educación ${ }^{5-7}$. Si bien hay un número de niños TEA con características más sutiles que se hacen evidentes en la edad escolar, muchos casos pueden diagnosticarse en forma confiable por profesionales médicos capacitados, alrededor de los 2 años ${ }^{8}$. El diagnóstico en varones es cuatro veces más frecuente que en niñas ${ }^{3}$, aunque el reconocimiento creciente de un fenotipo clínico menos marcado en niñas ha contribuido a reducir esta proporción' ${ }^{9}$. Las familias hispánicas que residen en EUA, se enfrentan a más dificultades para el acceso al diagnóstico y cuidados de salud que la población blanca, y los médicos generales consideran que los niños de estas familias son más difíciles de diagnosticar ${ }^{10}$. Daniels ${ }^{11}$ reportando resultados de una encuesta para cuidadores a 758 familias de países del sudeste europeo, muestra que, si bien los resultados varían entre países, existen insuficiencias en 
el diagnóstico oportuno y en el acceso y satisfacción a intervenciones terapéuticas.

En Chile, así como en otros países de América Latina, hay escasa información disponible acerca de las características de personas TEA y sus familias y de su acceso a diagnóstico oportuno, datos indispensables para que los niveles de decisión desarrollen políticas públicas efectivas dirigidas a este grupo ${ }^{12}$.

El presente estudio forma parte de un trabajo colaborativo de la Red de Espectro Autista Latinoamericano (REAL) compuesto por profesionales de Universidades e instituciones de seis países de Latinoamérica con el propósito de recabar información que permita una mejor comprensión de la situación y necesidades de las personas TEA en Chile y así contribuir al desarrollo de estrategias dirigidas a mejorar la calidad de vida de las personas TEA y sus familias. El objetivo de este trabajo es describir las características socio-demográficas y clínicas, primeras preocupaciones de los padres, edad de diagnóstico y profesional que lo realiza para aportar al conocimiento de la situación de las personas TEA y sus familias en Chile.

\section{Pacientes y Método}

\section{Desarrollo de la encuesta}

La Encuesta de Necesidades de los Cuidadores fue desarrollada por Autism Speaks en colaboración con la Iniciativa Mundial de Salud Pública para el Autismo (Global Autism Public Health Initiative (GAPH)), con el objetivo de evaluar las necesidades de las familias afectadas ${ }^{13}$. La encuesta está dirigida a padres o cuidadores de personas con un diagnóstico profesional de Trastorno del Espectro Autista (TEA), Trastorno Generalizado del Desarrollo (TGD), Trastorno Generalizado del Desarrollo No Especificado (TGDNE), Síndrome de Asperger, Autismo o Trastorno Autista; puede ser respondida por entrevista personal, telefónica unline y, toma un total aproximado de 30 minutos en ser completada.

La encuesta está compuesta por cuatro secciones y si el encuestado refiere tener más de un hijo TEA, se solicita responder en referencia al hijo mayor. La primera sección incluye información general y demográfica (relación con el niño, raza/etnia, educación, ciudad de residencia, etc.). La segunda incluye preguntas específicas relacionadas con las características de la persona TEA: edad, diagnóstico, primeras preocupaciones de los cuidadores, edad de diagnóstico y profesional que realizó el diagnóstico. Se solicitó a los respondentes estimar de acuerdo a categorías ofrecidas: a) el nivel de funcionamiento general actual de la persona TEA: compromiso leve, moderado o severo; b) habilidades verbales actuales: no verbal, palabras, frases de 2-3 palabras, oraciones de más de 4 palabras, oraciones complejas; c) Coeficiente Intelectual o Coeficiente de Desarrollo: retraso moderado/severo, retraso leve, promedio, sobre el promedio. Esta sección consideraba también preguntas relativas a qué profesional realizó el diagnóstico (pediatra, psicólogo, neurólogo, psiquiatra, médico de atención primaria, enfermera, equipo multidisciplinario, otro). La tercera sección aborda el uso/acceso de Servicios de Salud y Educación; se pide a los cuidadores que identifiquen cuáles servicios han usado alguna vez y cuáles usan actualmente. En la sección final se solicita la percepción de cuidadores/padres en cuanto a satisfacción con el acceso a servicios y las necesidades no cubiertas, el impacto de tener a una persona TEA para la familia y el cuidador, la percepción de estigma o auto-estigma y, por último, se incluyen preguntas acerca de calidad de vida. Las secciones 1 y 2 son el objeto de esta publicación.

\section{Implementación a nivel país}

La implementación de la encuesta estuvo a cargo de un coordinador nacional. Su trabajo contó con el apoyo y la asistencia técnica de la Red de Autismo Latinoamericana, la Organización Mundial de la Salud y Autism Speaks. Se consideró la traducción, un estudio piloto con 12 familias, traducción inversa, aprobación por el Comité de Ética y preparación de la encuesta para su amplia diseminación. La implementación incluyó además la selección de las fuentes de población a encuestar, el marco de muestreo, la difusión de las encuestas a las organizaciones o individuos receptores y la recopilación de las encuestas completadas.

Este estudio fue aprobado por el Comité de Ética de Investigación en Seres Humanos de la Facultad de Medicina, Universidad de Chile en conformidad con la Declaración de Helsinki. Inmediatamente antes de la encuesta se proporcionó en línea, un consentimiento informado y la descripción del estudio. La participación fue completamente voluntaria y anónima. No hubo compensación por la participación.

\section{Participantes}

La población objetivo fueron los cuidadores de personas TEA en Chile. Dada la falta de disponibilidad de registros de la población objetivo desde donde seleccionar individuos o unidades para el estudio, se utilizó un listado completo de proveedores de servicios, que incluía organizaciones que proveen cualquier tipo de intervenciones dirigidas a personas TEA. Se emplearon muchas otras fuentes para identificar a la población objetivo. Basados en la experiencia clínica, académica y de investigación de los autores, se generó una lista primaria de potenciales organizaciones, que se expandió a través de búsquedas en línea de información pública. Las organizaciones que cumplieron con los criterios de 
elegibilidad fueron contactadas por correo electrónico y recibieron la lista de contactos principales. Utilizando un enfoque de muestreo de bola de nieve, se pidió a los individuos que representaban a las organizaciones de la lista que recomendaran otras organizaciones no identificadas a través de la búsqueda primaria, hasta alcanzar la saturación.

La encuesta fue enviada por correo electrónico en todos los casos, completada en el hogar (90\%), en instituciones que prestan servicios de salud o educativos o en organizaciones de padres. Aproximadamente una quinta parte de los encuestados (21,9\%) necesitó ayuda para completar la encuesta.

\section{Resultados}

La muestra final estuvo compuesta por 291 participantes.

\section{Características de los cuidadores}

Las características de los cuidadores se muestran en tabla 1. La totalidad de los encuestados residen en Chile, siendo principalmente madres de individuos con autismo (86,3\%). En cuanto al nivel educacional, un 63,2\% de los participantes tiene Educación Superior. La mayoría de las familias residían en áreas urbanas: Región Metropolitana (incluyendo Santiago, ciudad capital y la más poblada $(49,8 \%)$, Valparaíso (10,8\%) y Antofagasta (7,2\%). El 15\% del grupo encuestado reportó tener al menos otro miembro de la familia nuclear con el diagnóstico de autismo.

Respecto del sistema de Salud utilizado, se informó que los proveedores de atención médica pertenecían al Sistema Público de Salud en 31,6\%, al Sistema de Salud Privado en 57\% o Institucional en 9,6\% (en Chile esta categoría comprende los Hospitales Institucionales de las Fuerzas Armadas y otras instituciones de carácter gremial, como es el Hospital del Profesor).

\section{Características de los individuos con autismo}

Las características de los individuos con autismo se muestran en la tabla 2. La mayoría eran varones (89\%) y su edad osciló entre 1 y 40 años (promedio: 10,44 DE: 6,05). La edad promedio al momento del diagnóstico fue de 58,0 meses (DE 36,5); la mediana fue de 46 meses.

Todos los individuos cumplieron alguna de las categorías de diagnóstico de TEA: trastorno del espectro autista (36,4\%), síndrome de Asperger (29,6\%) y trastorno generalizado del desarrollo $(14,8 \%)$. En cuanto a la estimación del nivel de déficit en el funcionamiento general (severidad del compromiso): éste se informó como grave en el 7,2\%, moderado en el 39,2\% y leve en el 39,9\%. El nivel de Lenguaje fue calificado como no verbal o con un nivel de lenguaje no superior a dos años, en aproximadamente un tercio de los individuos; un $12 \%$ fue calificado como capaz de expresarse con oraciones de más de cuatro palabras y el 53\% con oraciones complejas. En relación al nivel intelectual (CI) o de desarrollo psicomotor (CD) que les ha sido informado por los profesionales, los encuestados reportaron que un 53\% de los individuos tenía un CI/CD en el promedio o por encima de los rangos promedio; el 25,8\% reportó retraso leve, moderado o grave. Los problemas de conducta estaban presentes en el 61,2\% de los individuos.

\section{Primeras preocupaciones y diagnóstico}

Las primeras preocupaciones de los cuidadores surgieron a los 29,2 meses en promedio (DE 23,8). En $69 \%$ de los casos uno de los padres, principalmente la

\begin{tabular}{|c|c|}
\hline Relación con la persona TEA & N (\%) \\
\hline - Madre & $251(86,2)$ \\
\hline - Padre & $32(11,0)$ \\
\hline - Abuelos & $2(0,7)$ \\
\hline - Otros & $6(2,1)$ \\
\hline Nivel educacional del encuestado & N (\%) \\
\hline - Ninguna & $1(0,3)$ \\
\hline - Primaria incompleta & $1(0,3)$ \\
\hline - Primaria completa & $3(1,0)$ \\
\hline - Secundaria & $51(17,5)$ \\
\hline - Terciaria/nivel técnico & $51(17,5)$ \\
\hline - Grado profesional & $128(44,0)$ \\
\hline - Posgraduado & $56(19,2)$ \\
\hline Nivel educacional del otro padre & N (\%) \\
\hline - Ninguna & $2(0,7)$ \\
\hline - Primaria incompleta & $3(1,0)$ \\
\hline - Primaria completa & $3(1,0)$ \\
\hline - Secundaria & $57(19,6)$ \\
\hline - Terciaria/nivel técnico & $42(14,4)$ \\
\hline - Grado profesional & $126(43,3)$ \\
\hline - Posgraduado & $52(17,9)$ \\
\hline - No sabe & $6(2,1)$ \\
\hline Lugar de residencia & N (\%) \\
\hline - Región Metropolitana & $145(49,8)$ \\
\hline - Otras regiones & $133(45,7)$ \\
\hline - No responde & $13(4,5)$ \\
\hline Proveedores de salud & N (\%) \\
\hline - Salud pública & $92(31,6)$ \\
\hline - Institucional & $28(9,6)$ \\
\hline - Salud privada & $166(57,1)$ \\
\hline - Otro & $5(1,7)$ \\
\hline
\end{tabular}


madre, fue la primera persona en detectar características atípicas. Entre 18 posibles preocupaciones, los cuidadores mencionan 9 ( $\mathrm{DE} \pm 4,5$ ) en promedio; un $15 \%$ menciona cuatro o menos y la mitad de ellos menciona diez o más. En la figura 1 se observa la frecuencia con que cada preocupación fue reportada por los cuidadores. La preocupación más frecuente fue la dificultad para interactuar con otras personas $(79,4 \%)$. Otras preocupaciones presentes en más del 50\% de la muestra fueron: respuesta inusual a estímulos sensoriales $(69,8 \%)$, dificultades de comportamiento $(65,3 \%)$, gestos/movimientos inusuales $(64,3 \%)$, falta de contacto visual $(63,6 \%)$, intereses repetitivos $(58,4 \%)$, falta de comprensión de la comunicación no verbal $(56,4 \%)$, retraso de lenguaje $(55,3 \%)$, no hablaba como otros niños de la misma edad (50,9\%). Las preocupaciones menos mencionadas fueron problemas médicos $(17,5 \%)$, problemas de motricidad gruesa $(32,0 \%)$, dificultades para aprender cosas nuevas (alfabeto, números) $(32,8 \%)$, ausencia de habla $(35,1 \%)$, pérdida de habla u otras habilidades $(35,1 \%)$.

El diagnóstico fue realizado por un profesional de la salud en el 95\% de la muestra (figura 2). Los neurólogos pediátricos fueron los profesionales más frecuentemente mencionados en el diagnóstico de TEA (44,7\%), seguidos por los psiquiatras infantiles $(19,2 \%)$ y equipos de profesionales $(17,2 \%)$. Los pediatras fueron responsables del 5,5\% del total de los diagnósticos.

\section{Discusión}

Este estudio es el primer esfuerzo para evaluar sistemáticamente las características de personas TEA en nuestro país desde las percepciones de sus cuidadores. Los datos que se presentan proporcionan información valiosa acerca de las características de las personas TEA y sus familias, de la detección por sus padres de dificultades en el desarrollo y de la edad y características del diagnóstico. Esta información constituye una base para el desarrollo de estrategias y políticas de salud y educación.

En esta muestra de conveniencia, los cuidadores muestran un sesgo hacia un grupo socioeconómico por encima del promedio chileno en muchas de las variables descriptivas como: una mayor proporción de familias urbanas versus rurales ${ }^{14}$; un nivel educativo superior con un $60 \%$ de cuidadores que informan un título universitario o superior, frente al $21 \%$ de la población chilena ${ }^{15}$; un porcentaje de personas adscritas a Sistema Privado de Salud de 57\% frente al 20\% de la población chilena ${ }^{16}$. Además, los factores mencionados permiten esperar un mejor uso de las herramientas digitales, que facilitan completar adecuadamente esta encuesta. A la inversa, otros cuidadores tendrían mayores
Tabla 2. Características de 291 personas trastornos del espectro autista (TEA), reportadas por sus cuidadores

\begin{tabular}{|c|c|}
\hline Sexo & N (\%) \\
\hline - Varón & $259(89,0)$ \\
\hline - Mujer & $32(11,0)$ \\
\hline Edad (años), (promedio (DE)) & $10,44(6,05)$ \\
\hline Rango (años) & $1-40$ \\
\hline $\begin{array}{l}\text { Diagnóstico actual } \\
\text { - Trastorno espectro autista } \\
\text { - Trastorno generalizado desarrollo } \\
\text { - Síndrome Asperger } \\
\text { - Autismo/trastorno autista } \\
\text { - Trastorno generalizado desarrollo no especificado }\end{array}$ & $\begin{array}{c}N(\%) \\
106(36,4) \\
43(14,8) \\
86(29,6) \\
28(9,6) \\
28(9,6)\end{array}$ \\
\hline $\begin{array}{l}\text { Nivel de funcionamiento general actual } \\
\text { - Compromiso leve } \\
\text { - Compromiso moderado } \\
\text { - Compromiso severo } \\
\text { - No sabe } \\
\text { - No responde }\end{array}$ & $\begin{array}{l}N(\%) \\
116(39,9) \\
114(39,2) \\
21(7,2) \\
29(10,0) \\
11(3,8)\end{array}$ \\
\hline $\begin{array}{l}\text { Habilidades verbales actuales } \\
\text { - No verbal } \\
\text { - Palabras } \\
\text { - Frases de 2-3 palabras } \\
\text { - Oraciones > } 4 \text { palabras } \\
\text { - Oraciones complejas } \\
\text { - No responde }\end{array}$ & $\begin{array}{c}N(\%) \\
38(13,1) \\
25(8,6) \\
35(12,0) \\
35(12,0) \\
155(53,3) \\
3(1,0)\end{array}$ \\
\hline $\begin{array}{l}\text { Estimación } \mathrm{Cl} / \mathrm{CD} \text { * } \\
\text { - Retraso moderado/severo } \\
\text { - Retraso leve } \\
\text { - Promedio } \\
\text { - Sobre el promedio } \\
\text { - No sabe } \\
\text { - No responde }\end{array}$ & $\begin{array}{c}N(\%) \\
29(10,0) \\
46(15,8) \\
88(30,2) \\
68(23,4) \\
50(17,2) \\
10(3,4)\end{array}$ \\
\hline Problemas conductuales actuales & $\mathrm{N}(\%)$ \\
\hline Sí & $178(61,2)$ \\
\hline No & $113(38,8)$ \\
\hline Edad de primeras preocupaciones (meses), promedio (DE) & $29,2(23,8)$ \\
\hline Edad de diagnóstico (meses), promedio (DE) & $58,0(36,5)$ \\
\hline
\end{tabular}

dificultades para acceder o completar la encuesta por menor experiencia con sistemas digitales. En consecuencia, la información recogida refleja posiblemente una realidad ventajosa en comparación a la situación de la mayoría de las familias de nuestro país.

Es necesario hacer una nota de precaución en relación a la ventana de tiempo de los datos recopilados, dado el amplio rango de edades de las personas TEA de las familias encuestadas. La proporción de hombres versus mujeres de 8,9:1,1 mayor que lo reportado, pueden atribuirse a sub-diagnóstico de TEA en mujeres, 


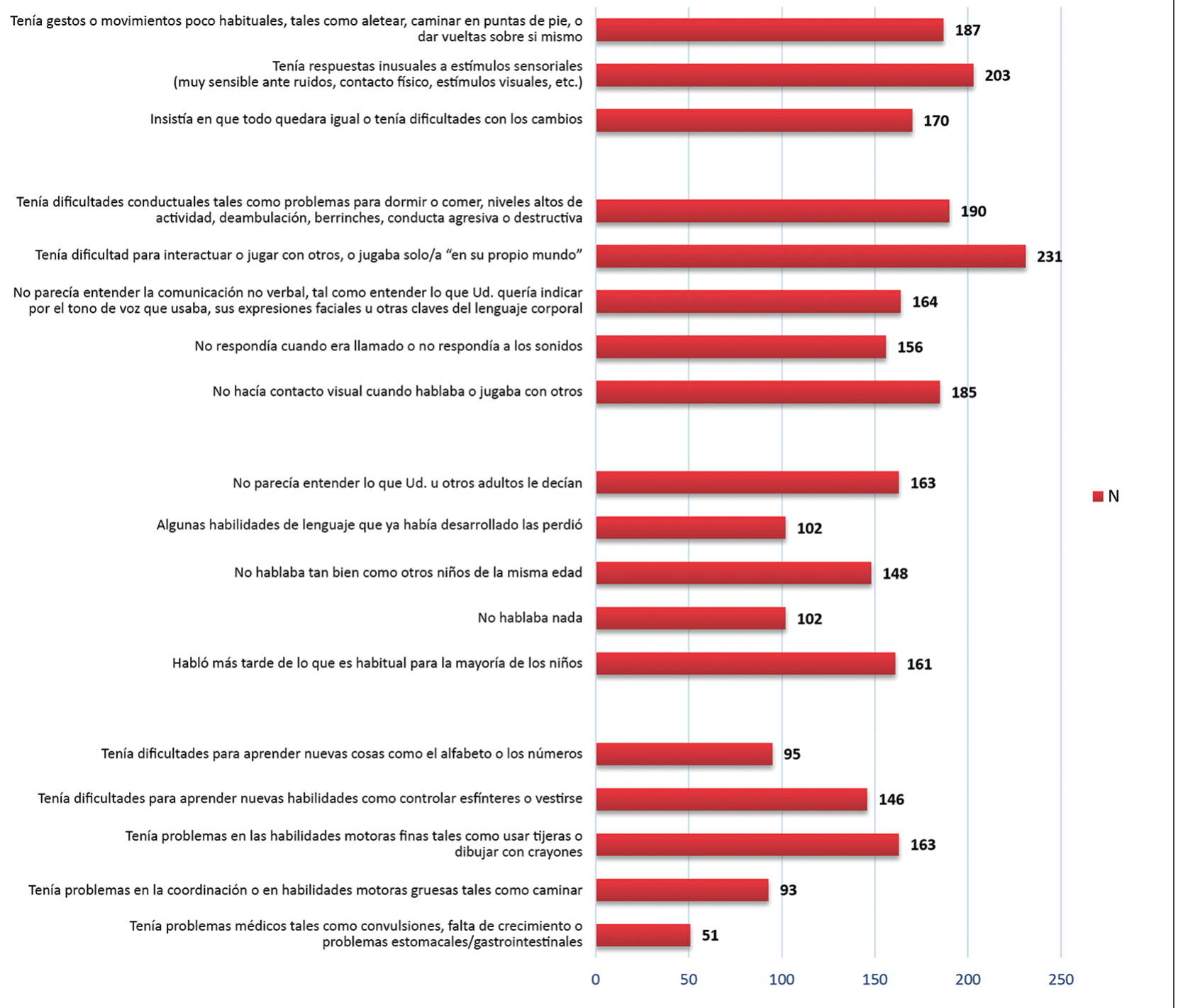

Figura 1. Naturaleza y frecuencia de primeras preocupaciones reportadas por cuidadores, en 291 personas TEA.

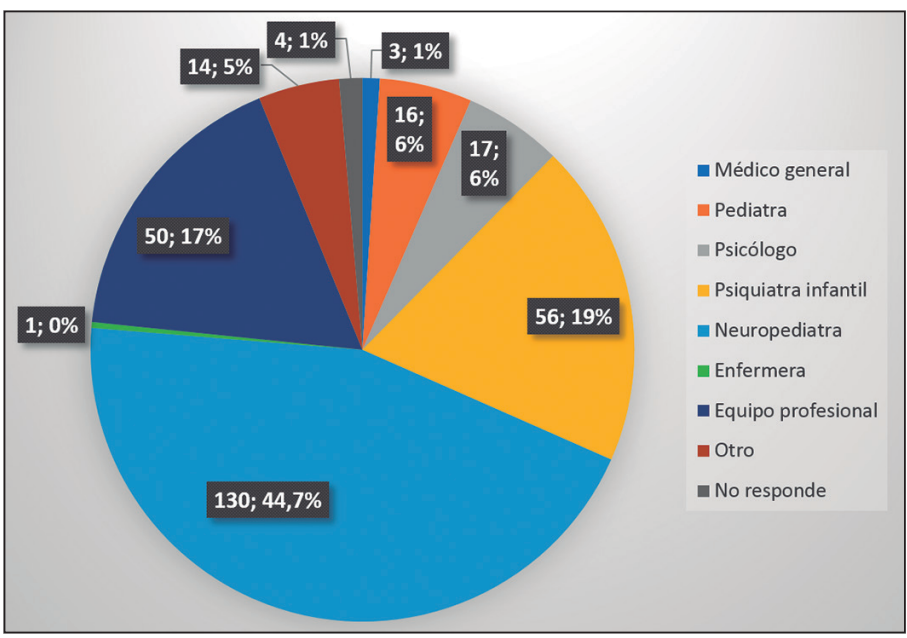

Figura 2. Profesional que diagnosticó trastorno espectro autista en 291 personas encuestadas. situación que se ha revertido en los últimos años, por el reconocimiento de una expresión diferente y sutil de la condición dependiendo del género ${ }^{9}$. Las variaciones observadas en las categorías de diagnóstico obedecen a la evolución temporal de los conceptos de diagnóstico y clasificación ${ }^{1,11,17}$.

El nivel de funcionamiento general, de lenguaje $y$ de CI/CD reportados en esta muestra, con un $40 \%$ $50 \%$ de los individuos con deficiencias menores de lenguaje, CI promedio y sólo deficiencias leves en el funcionamiento, es similar a lo reportado en estudios epidemiológicos recientes en EE. UU. de Norteamérica ${ }^{3}$ y posiblemente se relaciona con el sesgo socioeconómico y cultural de esta muestra. Por otra parte, la prevalencia de discapacidad intelectual en TEA, muestra una tendencia a disminuir en el tiempo. Las estimaciones previas de 70\%-75\%, se han reducido gradualmente, 
siendo actualmente de alrededor de un $30 \%-40 \%$, con un 20\%-30\% de CI limítrofes y alrededor de 40\%-50\% en el rango normal. Esta tendencia es atribuible en parte importante a un mejor reconocimiento de la condición y a la inclusión de una mayor amplitud fenotípica, con casos levemente afectados y/o con un mejor funcionamiento adaptativo ${ }^{18}$.

Uno de los hallazgos más importantes de esta encuesta es la tardía edad de diagnóstico, de 58 meses, con un intervalo transcurrido en promedio mayor de dos años entre la primera preocupación de los padres y el momento del diagnóstico. A los 24 y 36 meses, un $59 \%$ y $79 \%$ respectivamente, de los cuidadores informaron haber tenido una preocupación importante por el desarrollo de su hijo, sin embargo, sólo el 12,2\% y el $37,4 \%$ de los niños habían sido diagnosticados hasta esos momentos. Esta preocupante tardanza en identificar a niños y niñas TEA también se observa y subsiste en países desarrollados como EUA, Reino Unido y Canadá, a pesar de la mayor sensibilización en la identificación de signos tempranos, donde la edad de diagnóstico se mantiene entre 4 y 5 años con una tendencia temporal hacia una identificación más temprana ${ }^{7}$. La gravedad de los síntomas, el mejor nivel socio-económico, la mayor preocupación de los padres y las interacciones previas con los sistemas de salud y educación son factores que han mostrado una asociación consistente con un diagnóstico más temprano. Una publicación reciente de los resultados de esta encuesta en el sudeste de Europa a 789 cuidadores, incluidos Albania, Bulgaria, Croacia y Turquía, reveló una edad promedio de primeras preocupaciones de 24,4 meses, muy similar a la encuesta chilena, sin embargo, la edad del diagnóstico fue más temprana, de 40 meses. Esta diferencia con nuestro estudio podría atribuirse a una muestra más joven y de menor nivel de funcionamiento ${ }^{11}$. Montiel ${ }^{19}$ informa sobre el único estudio de niños TEA en América Latina, que similar a lo señalado muestra que las preocupaciones de los padres aparecen tempranamente a los 17 meses, pero el diagnóstico se demora 3 años en promedio (53-54 $\mathrm{m})$. Salomone ${ }^{20}$ estudiando una muestra de 23 países europeos, compuesta por 1.410 niños menores de 7 años, encontró una edad de diagnóstico de 42 meses, con una variación considerable entre países, que va desde los 33-50 meses. Los niños con mejor capacidad verbal se identificaron más tarde, especialmente si eran mujeres, apoyando las nociones de una manifestación más sutil en mujeres y que los niños con síntomas más leves se diagnostican más tarde $e^{7,11,19-21}$.

En el presente estudio, la edad de primeras preocupaciones de los padres fue en promedio dos años antes del diagnóstico, alrededor de los 30 meses de vida, cifra más alta que lo reportado en la literatura ${ }^{11,22,23}$. El porcentaje de padres que reconocen alguna alteración del desarrollo antes de la edad de 1 año $(18,2 \%)$ y antes de la edad de dos años (59\%) también es menor a lo observado en otros estudios. Publicaciones, provenientes de diferentes diseños y poblaciones de estudio, informan un promedio de rango de edad de las primeras preocupaciones entre 14 y 24 meses, con aproximadamente un tercio de los padres reportando primeras preocupaciones a la edad de un año, y tres cuartos a los 2 años $^{11,22-28}$. El retraso en el reconocimiento de las dificultades, observado en esta muestra, señalaría una menor conciencia de los hitos del desarrollo temprano en el área social y emocional, específicamente de los signos de alerta de autismo, comparativamente con otros logros del desarrollo como son los hitos el desarrollo motor.

Es de la mayor relevancia destacar que solamente en un 5\% de esta muestra el diagnóstico fue hecho por el pediatra. Considerando que la consulta pediátrica es un nodo crítico para el reconocimiento y diagnóstico oportuno de TEA y los pediatras los primeros profesionales a quienes los padres refieren sus preocupaciones, son éstos los llamados a proveerlos de orientación confiable. Su capacidad de respuesta es determinante para iniciar un adecuado proceso diagnóstico y terapéutico. En oposición, la pérdida de esta oportunidad privilegiada para sospechar o realizar el diagnóstico, significa desaprovechar una ventana terapéutica irrecuperable que incidirá decisivamente en el pronóstico a largo plazo. Algunos estudios muestran que factores como la falta de un entrenamiento adecuado en trastornos del desarrollo o reticencia a agobiar a los padres validando su preocupación, los hacen adoptar una conducta pasiva $^{29}$. Llegar al diagnóstico de TEA es a menudo un proceso largo y difícil que involucra a muchos actores: el niño, su familia, el pediatra, especialistas y terapeutas, entre otros. El clínico tiene un rol fundamental en entregar a los padres una idea clara del proceso que implica obtener un diagnóstico de TEA, ayudarlos a ampliar las redes sociales y orientarlos para una interacción fluida con las estructuras de atención existentes asociadas a los TEA ${ }^{30,31}$.

Dentro de las limitaciones de este estudio destaca que se trata de una muestra de conveniencia, que en este caso tiene un sesgo hacia un grupo con un nivel socioeconómico más alto que la población chilena en general y por lo tanto refleja "la mejor situación" de las personas TEA en Chile. Por otro lado, la amplia ventana de tiempo que se recoge, en una muestra que incluye desde bebés hasta adultos, pone en duda el efecto de los cambios en el tiempo que influyen en muchas, si no en todas, las variables estudiadas. A la vez, corresponde mencionar que la confiabilidad de esta información es limitada, por tratarse de una declaración hecha por los encuestados que no es verificable. La información recogida proviene de los cuidadores, lo que no permite 
confirmar con certeza los diagnósticos o el estado actual de funcionamiento y adaptación de las personas TEA. Se ha señalado que las estimaciones de los padres sobre los niveles cognitivos de sus niños TEA, parecen ser más inexactas en los niveles más altos de funcionamiento ${ }^{18}$.

Estos resultados pueden orientar otras investigaciones, centrándose en áreas prioritarias para establecer políticas y acciones de salud en esta población. Uno de los más inmediatos es el diagnóstico precoz y la reducción de la brecha de tiempo entre las primeras preocupaciones de los cuidadores y el diagnóstico, en los servicios relacionados con la infancia y la adolescencia. En futuros estudios será necesario además buscar nuevas metodologías de recolección de información y datos para los sectores más desfavorecidos de la sociedad (rural, bajo nivel educativo, etc.) para conocer la realidad de manera transversal. También surge la necesidad de hacer estudios por grupo etario: menores de dos años, 2 a 5 años, 6 a 12 años, 12 a 18 y mayores de 18 años dadas las diferentes características evolutivas y de servicios que se brindan en el país.

Chile es un país con una larga tradición de salud pública. La atención al crecimiento y desarrollo infantil han sido áreas de preocupación prioritarias. Sin embargo, el énfasis del monitoreo se ha puesto en el desarrollo psicomotor y en hitos cognitivos específicos, no así en aspectos relacionados con el desarrollo socioemocional y comunicacional. Sólo recientemente, el problema del TEA se ha erigido como un tema relevante para la sociedad y medios de comunicación, introduciéndose gradualmente en los programas de salud y en los currículos académicos de las carreras de ésta área. Los datos recogidos en esta investigación son extraordinariamente importantes de considerar en las diferentes formas de atención, requerimientos y situación de salud mental de las personas TEA.

\section{Conclusiones}

Este es el primer estudio exploratorio descriptivo de cuidadores, generalmente padres y madres, de personas TEA en Chile. La situación de las personas TEA en Chile es más desfavorable que lo observado en estudios similares realizados en países del sur de Europa ${ }^{11}$ en términos de características, edad de primeras preocupaciones y retraso en el diagnóstico.

La muestra que respondió a esta encuesta en Chile está conformada principalmente por cuidadores de mayor nivel socio-económico y educacional, con mayor acceso a medios digitales. Aun así, los resultados del presente estudio muestran indicadores importantes como el retraso en el diagnóstico clínico de cerca de dos años y medio a contar de la primera preocupación de los padres, generando menos oportunidades para el desarrollo global de estos niños y comprometiendo su pronóstico posterior. Es posible que los sistemas de Salud y Educación, no estén dando una respuesta adecuada al diagnóstico precoz, hecho que se representa en la escasa participación de los pediatras en la detección oportuna de TEA.

\section{Responsabilidades Éticas}

Protección de personas y animales: Los autores declaran que los procedimientos seguidos se conformaron a las normas éticas del comité de experimentación humana responsable y de acuerdo con la Asociación Médica Mundial y la Declaración de Helsinki.

Confidencialidad de los datos: Los autores declaran que han seguido los protocolos de su centro de trabajo sobre la publicación de datos de pacientes.

Derecho a la privacidad y consentimiento informado: Los autores han obtenido el consentimiento informado de los pacientes y/o sujetos referidos en el artículo. Este documento obra en poder del autor de correspondencia.

\section{Conflicto de intereses}

Los autores declaran no tener conflicto de intereses.

\section{Agradecimientos}

A las familias y cuidadores que accedieron a participar en esta encuesta. 


\section{Referencias}

1. Maenner MJ, Shaw KA, Baio J, et al. Prevalence of Autism Spectrum Disorder Among Children Aged 8 Years-Autism and Developmental Disabilities Monitoring Network, 11 Sites, United States, 2016 [published correction appears in MMWR Morb Mortal Wkly Rep. 2020;69(16):503]. MMWR Surveill Summ. 2020;69(4):1-12. Published 2020 Mar 27. doi:10.15585/mmwr.ss6904a1).

2. Jensen CM, Steinhausen HC, Briciet Lauritsen M. Time Trends Over 16 Years in Incidence-Rates of Autism Spectrum Disorders Across the Lifespan Based on Nationwide Danish Register Data. J Autism Dev Disord. 2014;44:180818.

3. Davidovitch M, Hemo B, ManningCourtney P, Fombonne E. Prevalence and Incidence of Autism Spectrum Disorder in an Israeli Population. J Autism Dev Disord. 2013;43:785-93.

4. Baio J, Wiggins L, Christensen DL, et al. Prevalence of Autism Spectrum Disorder Among Children Aged 8 Years - Autism and Developmental Disabilities Monitoring Network, 11 Sites, United States, 2014. MMWR Surveill Summ. 2018;67:1-23.

5. Zwaigenbaum L, Bauman ML, Fein D, et al. Early Screening of Autism Spectrum Disorder: Recommendations for Practice and Research. Pediatrics. 2015; 136:S4159.

6. Zwaigenbaum L, Bauman ML, Choueiri $\mathrm{R}$, et al. Early Intervention for Children With Autism Spectrum Disorder Under 3 Years of Age: Recommendations for Practice and Research. Pediatrics. 2015;136:S60-81.

7. Benevides TW, Carretta HJ, Mandell DS. Differences in Perceived Need for Medical, Therapeutic, and Family Support Services among Children with ASD. Pediatrics. 2016;137:S176-85.

8. Daniels AM, Mandell DS. Explaining differences in age at autism spectrum disorder diagnosis: a critical review. Autism. 2014;18:583-97.

9. Guthrie W, Swineford LB, Nottke C, Wetherby AM. Early diagnosis of autism spectrum disorder: Stability and change in clinical diagnosis and symptom presentation. J Child Psychol Psychiatry 2013;54(5):582-90.

10. Loomes R, Hull L, Mandy WPL. What is the male-to-female ratio in autism spectrum disorder? A systematic review and meta-analysis. J Am Acad Child Adolesc Psychiatry 2017;56(6):46674.

11. Zuckerman KE, Sinche B, Mejía A, Cobian M, Becker T, Nicolaidis C. Latino parents' perspectives on barriers to autism diagnosis. Acad Pediatr. 2014;14:301-8.

12. Daniels AM, Como A, Hergüner $S$, Kostadinova K, Stosic J, Shih A. Autism in Southeast Europe: A Survey of Caregivers of Children with Autism Spectrum Disorders. J Autism Dev Disord. 2017;47:2314-25.

13. Baxter AJ, Brugha TS, Erskine HE, Scheurer RW, Vos T, Scott JG. The epidemiology and global burden of autism spectrum disorders. Psychol Med. 2015;45:601-13.

14. Autism Speaks. (2016). Autism Speaks Global Public Health Initiative website. Accessed June 11, 2016 from: https:// www.autismspeaks.org/science/researchinitiatives/global-autism-public-health.

15. Instituto Nacional de Estadísticas: Síntesis de resultados Censo 2017. 2018.https:// www.censo2017.cl/descargas/home/ sintesis-de-resultados-censo2017.pdf.

16. Delpiano A, Quiroga V, Díaz MI. Análisis de indicadores educativos de Chile y la OCDE en el contexto de la Reforma Educacional. Ser Evidencias. 2015;31:19. http://centroestudios.mineduc.cl/ tp_enlaces/portales/tp5996f8b7cm96/ uploadImg/File/Evidencias/ Evidencias\%20final_noviembre_2015.pdf.

17. Aravena P, Inostroza M. ¿Salud pública o privada? Los factores más importantes al evaluar el sistema de salud en Chile. Rev Med Chile. 2015;143:244-51.

18. Volkmar FR, McPartland JC. From Kanner to DSM-5: Autism as an Evolving Diagnostic Concept. Annu Rev Clin Psychol. 2013;10:193-212.

19. Chakrabarti S, Fombonne E. Pervasive developmental disorders in preschool children: confirmation of high prevalence. Am J Psychiatry. 2005; 162(6):1133-41.

20. Montiel-Nava C, Chacín JA, GonzálezÁvila Z. Age of diagnosis of autism spectrum disorder in Latino children:
The case of Venezuelan children. Autism. 2017;21:573-80.

21. Salomone E, Charman T, Mcconachie H, Warreyn P. Child's verbal ability and gender are associated with age at diagnosis in a sample of young children with ASD in Europe. Child Care Health Dev. 2016;42:141-5.

22. Sheldrick RC, Maye MP, Carter AS. Age at First Identification of Autism Spectrum Disorder: An Analysis of Two US Surveys. J Am Acad Child Adolesc Psychiatry. 2017;56:313-20.

23. Howlin P, Moore A. Diagnosis in autism: A survey of over 1200 patients in the UK. Autism. 1997;1:135-62.

24. Zablotsky B, Colpe LJ, Pringle BA, Kogan MD, Rice C, Blumberg SJ. Age of parental concern, diagnosis, and service initiation among children with autism spectrum disorder. Am J Intellect Dev Disabil. 2017;122:49-61.

25. Herlihy L, Knoch K, Vibert B, Fein D. Parents' first concerns about toddlers with autism spectrum disorder: Effect of sibling status. Autism. 2015;19:20-8.

26. De Giacomo A, Fombonne E. Parental recognition of developmental abnormalities in autism. Eur Child Adolesc Psychiatry. 1998;7:131-6.

27. Matheis M, Matson JL, Burns CO, et al. Factors related to parental age of first concern in toddlers with autism spectrum disorder. Dev Neurorehabil. 2017;20:22835.

28. Chawarska K, Paul R, Klin A, Hannigen S, Dichtel LE, Volkmar F. Parental recognition of developmental problems in toddlers with autism spectrum disorders. J Autism Dev Disord. 2007;37:62-72.

29. Baghdadli A, Picot MC, Pascal C, Pry R, Aussilloux C. Relationship between age of recognition of first disturbances and severity in young children with autism. Eur Child Adolesc Psychiatry. 2003;12:122-7.

30. Carbone PS, Norlin C, Young PC. Improving Early Identification and Ongoing Care of Children with Autism Spectrum Disorder. Pediatrics. 2016;137(6). pii: e20151850.

31. Lappé M, Lau L, Dudovitz RN, Nelson BB, Karp EA, Kuo AA. The Diagnostic Odyssey of Autism Spectrum Disorder. Pediatrics. 2018;141(Suppl 4):S272-9. 